Volume 2

\title{
Does the Expression of Ki-67, p16 and COX-2 at Initial Diagnosis of Breast Atypia or Usual Ductal Hyperplasia Predict a Second Clinically Significant Event?
}

\author{
Judy A. Tjoe \\ Philippe Gascard \\ Jianxin Zhao \\ Gary F. Neitzel \\ Maharaj Singh \\ Brittany Last \\ James Marx \\ Thea Tlsty \\ Sanjay Kansra
}

Follow this and additional works at: https://aah.org/jpcrr

Part of the Biological Phenomena, Cell Phenomena, and Immunity Commons, Neoplasms Commons, Oncology Commons, and the Surgery Commons

\section{Recommended Citation}

Tjoe JA, Gascard P, Zhao J, Neitzel GF, Singh M, Last B, Marx J, Tlsty T, Kansra S. Does the Expression of Ki-67, p16 and COX-2 at Initial Diagnosis of Breast Atypia or Usual Ductal Hyperplasia Predict a Second Clinically Significant Event?. J Patient Cent Res Rev 2015;2:215-216. http://dx.doi.org/10.17294/

2330-0698.1246

Published quarterly by Midwest-based health system Advocate Aurora Health and indexed in PubMed Central, the Journal of Patient-Centered Research and Reviews (JPCRR) is an open access, peer-reviewed medical journal focused on disseminating scholarly works devoted to improving patient-centered care practices, health outcomes, and the patient experience. 
mass index $48.62 \pm 8.0 \mathrm{~kg} / \mathrm{m}^{2}$ and mean preoperative excess weight $171.28 \pm 52.0 \mathrm{lbs}$. Median follow-up after LAGB was 63.63 months (range $0.0-162.4$ ) for a total of 4,859 patientyears. During follow-up, 103 patients (11.9\%) underwent reoperation for slippage at a median of 54.26 months (range 0.0-160.50) after LAGB. We found a significantly lower weight at rebanding, and at 1, 2, 3, 4 and 5 years after rebanding in patients with slippage compared to their initial weight, and their weight at 1, 2, 3, 4 and 5 years after LAGB. There was a significantly lower excess weight loss failure rate in patients with slippage compared to matching controls ( $40 \%$ vs. $60 \%, \mathrm{P}=0.0006)$ after first year. There were no differences in EWL rate between the two groups after first year. In multivariate analysis only female gender was significantly associated with slippage.

Conclusion: Failure rate of excess weight loss after rebanding for slippage was lower or similar to the failure rate after initial laparoscopic adjustable gastric banding.

\section{Feasibility of Atrial Delivery and Tracking of Stem Cells in a Porcine Model}

Nina Garlie, Timothy Hacker, Eric G. Schmuck, Jill Koch, Jayant Khitha, Amish Raval, Indrajit Choudhuri

\section{Regenerative Medicine Center, Aurora Research Institute, Aurora Health Care; Department of Medicine, University of Wisconsin School of Medicine and Public Health; Aurora Cardiovascular Services, Aurora Health Care}

Background: Many patients undergoing open heart surgery have sinus node dysfunction and atrial fibrillation, leading to adverse outcomes. Mesenchymal stem cells (MSC) delivered at the time of surgery may have a reparative effect on atrial tissue, thereby improving sinus node function and reducing or preventing atrial fibrillation. Stem cell delivery to the atrium is entirely unstudied. This is a significant gap in medical research, as atrial disease contributes significantly to health care costs.

Purpose: The purpose of this pilot study is to establish a technique to deliver MSC to the atria through an open-chest model, to assess the safety of this technique, and to evaluate the acute retention of the delivered cells.

Methods: All in vivo animal experimentation was approved by the University of Wisconsin Animal Care and Use Committee and took place in the Cardiovascular Physiology Core Facility at UW-Madison. MSC (3-5×106 in $50 \mu 1$ per site) were injected intramyocardially during an open-chest procedure in anesthetized pigs. To track the cells in vivo, MSC were labeled with $18 \mathrm{FDG}$ then visualized at 1 and 6 hours postinjection by PET/CT. Pigs were monitored for intraoperative arrhythmia, bleeding and hypotension.

Results: By gently repositioning the heart, both atria were accessible for the injections. The thickest part of each atrium was isolated and stabilized briefly for the injection using a hemostat. The injected cells were visible by PET/CT 1 and 6 hours postinjection. However, when the MSC were labeled with $10 \mathrm{mCi} 18 \mathrm{FDG}$, the signal was too high, causing a bloom around the areas of injection. So the dose was lowered to $5 \mathrm{mCi} 18 \mathrm{FDG}$, which resulted in a clear signal at 1 hour in both atria. At 6 hours, the right atrial injection was still easy to read, but the left injection was difficult to resolve from background signal. All injections resulted in cell leakage from the injection site and uptake of the signal into the lungs. However, pulmonary function as measured by $\mathrm{SpO}_{2}$ and $\mathrm{EtCO}_{2}$ was unchanged. Intraoperative arrhythmias detected during the injections were caused by manipulation of the heart. No additional arrhythmias were detected. No bleeding or hypotension was observed as a result of the injections.

Conclusion: This pilot study demonstrated that atrial delivery of MSC is feasible and safe in an open-chest porcine model and that MSC are retained for at least 6 hours postinjection. Subsequent studies will determine the ability of MSC to downregulate inflammation, decrease scarring and prevent sinus node dysfunction.

\section{Does the Expression of Ki-67, p16 and COX-2 at Initial Diagnosis of Breast Atypia or Usual Ductal Hyperplasia Predict a Second Clinically Significant Event?}

Judy A. Tjoe, Philippe Gascard, Jianxin Zhao, Gary F. Neitzel, Maharaj Singh, Brittany Last, James Marx, Thea Tlsty, Sanjay Kansra

Surgical Breast Oncology, Aurora Health Care; Department of Pathology, University of California, San Francisco; ACL Laboratories, Department of Pathology, Aurora Health Care; Aurora Research Institute, Aurora Health Care

Background: Women diagnosed with atypical ductal hyperplasia $(\mathrm{ADH})$ or atypical lobular hyperplasia (ALH) have a fivefold increased risk of developing breast cancer. Because ADH/ALH can be precursors or predictive markers of a subsequent clinically significant event (SCSE), i.e. atypia, in situ or invasive carcinoma, the clinical outcome for these patients ranges anywhere from remission to invasive malignancy. Currently we cannot predict which atypical breast lesion is likely to be associated with future cancer, resulting in aggressive management and, possibly, overtreatment. Kerlikowske et al. reported that a combination of three biomarkers (cell cycle regulator p16INK4a, proliferation antigen Ki-67 and stress response enzyme COX-2) predicted risk of progression for $\sim 50 \%$ of women diagnosed with ductal carcinoma in situ and treated by lumpectomy alone.

Purpose: To evaluate whether expression levels of p16, Ki-67 and COX-2 predict risk of development of a SCSE in patients initially diagnosed with breast atypia (ADH or ALH) or usual ductal hyperplasia (UDH).

Methods: Patients with an initial diagnosis of pure $\mathrm{ADH} / \mathrm{ALH}$ were identified by medical record review and the lesion confirmed by a single pathologist blinded 
to original diagnosis. Twelve women who developed a SCSE (cases) were matched to 44 women who did not (controls) at least 5 years after initial diagnosis. Archived tissues were stained for p16INK4a, Ki67 and COX-2 using "multiplex immunohistochemistry," enabling simultaneous interrogation of expression levels of the three biomarkers in a single tissue section.

Results: Our multiplex analysis revealed that expression levels of Ki-67, p16INK4a or COX-2, either in epithelial cells within the lesion or in stromal cells adjacent to the lesion, either individually or in combination, do not predict the development of a SCSE in women initially diagnosed with $\mathrm{ADH} / \mathrm{ALH}$ or UDH. However, this analysis identified double- or triple-positive cells in the vicinity of the lesions in some cases and controls.

Conclusion: Expression of Ki-67, p16INK4a and COX-2 is not predictive of a SCSE following initial diagnosis of ADH/ ALH or UDH. Further analysis is needed on a larger cohort after longer follow-up after initial diagnosis to confirm our findings and to investigate whether the presence of doubleor triple-positive cells (a signature predicted to correlate with poor outcome) is predictive of progression regardless of the expression status of the lesion.

\section{The Effect of Percutaneous Closure of Large Atrial Septal Defects on Right Ventricular Function in Adults}

Armaan Shaikh, Alejandro Lopez-Mas, Suhail Allaqaband, Bijoy K. Khandheria, Abraham Getenet, Matt M. Umland, Maharaj Singh, Tanvir Bajwa

Aurora Cardiovascular Services, Aurora Health Care; Department of Internal Medicine, Aurora Sinai Medical Center; Aurora Research Institute, Aurora Health Care

Background: Percutaneous closure of atrial septal defects has been shown to be a safe alternative to surgery. Despite this, past studies have largely been focused on either smallto medium-sized atrial septal defects or percutaneous closure in children and young adults.

Purpose: Our study sought to examine if right ventricular function and size improved after percutaneous closure of large atrial septal defects in the adult population.

Methods: Over a 5-year span, 25 patients underwent percutaneous closure of a secundum atrial septal defect with an occluder device. A retrospective examination was conducted for each patient, including both echocardiography and chart review for postdevice complications/symptoms.

Results: Average patient age was 44.4 years, and mean device size was $28 \mathrm{~mm}$ (range: $24-38 \mathrm{~mm}$ ). Follow-up echocardiography (mean of 134 days) showed tricuspid annular plane systolic excursion was significantly improved (2.11 vs. $2.33 ; \mathrm{P}=0.013)$. There also was a significant reduction in right ventricular diastolic chamber size (31.0 vs. 35.4; $\mathrm{P}<0.01$ ). At 1 -year postprocedure follow-up, zero patients had experienced transient ischemic attack, stroke or device perforation/embolization.
Conclusion: Percutaneous closure of large secundum atrial septal defects in adults improves right ventricular function as well as right ventricular chamber size. Percutaneous closure of large atrial septal defects also is a safe, very low-risk procedure in terms of future adverse neurologic, embolic or perforation-related events.

\section{Coronary Aorta Systolic and Diastolic Pressure Indices: Two Novel Indicators for Predicting Significant Coronary Stenosis - A Validation Against Fractional Flow Reserve}

Mirza Mujadil Ahmad, Khawaja Afzal Ammar, Mirza Nubair Ahmad, Arsalan Riaz, Fatima A. Husain, Syed Shahab Kazmi, Imran Husain, Anjan Gupta

\section{Aurora Cardiovascular Services, Aurora Health Care}

Background: Since most of the coronary flow occurs in diastole, either mean $\mathrm{Pd} / \mathrm{Pa}$ or iFR has been used to measure the hemodynamic significance of a coronary stenosis. We have observed that a significant pressure gradient exists in coronary stenosis even in systole, which is contrary to general understanding but similar to ankle brachial index. Furthermore, prior studies have evaluated baseline $\mathrm{Pd} / \mathrm{Pa}$ (mean coronary artery/mean aorta pressure) ratio as well as iFR (instantaneous wave-free ratio obtained during entire period of diastole) to predict fractional flow reserve (FFR) $\leq$ 0.80 . We hypothesized a simple end-systolic and -diastolic pressure measurement in the coronary artery distal to stenosis may perform adequately to predict FFR, obviating a need to measure $\mathrm{Pd} / \mathrm{Pa}$ or $\mathrm{iFR}$.

Purpose: We sought to evaluate systolic and diastolic $\mathrm{Pd} /$ $\mathrm{Pa}$, and termed them coronary artery systolic pressure index (CASPI) and coronary artery diastolic pressure index (CADPI), respectively, against $\mathrm{FFR} \leq 0.80$.

Methods: After retrospectively identifying 555 moderate stenotic lesions undergoing FFR measurement at a tertiary care center over a 4-year period, we procured original pressure tracings obtained during the cardiac catheterization and manually measured systolic and diastolic pressures in the aorta and in the coronary artery distal to the stenosis, before and after adenosine infusion. Utilizing FFR $\leq 0.80$, operating test characteristics of CASPI and CADPI were measured and compared to those of baseline $\mathrm{Pd} / \mathrm{Pa}$.

Results: In the 555 lesions, mean CASPI $(0.97 \pm 0.04)$ and CADPI $(0.95 \pm 0.08)$ were similar to baseline $\mathrm{Pd} / \mathrm{Pa}(0.95 \pm$ 0.05 ). CASPI correlated well with baseline $\mathrm{Pd} / \mathrm{Pa}$ (Spearman $\mathrm{r}=0.81 ; \mathrm{P}<0.0001)$. Similarly, CADPI was strongly correlated with baseline $\mathrm{Pd} / \mathrm{Pa}(0.86 ; \mathrm{P}<0.0001)$. The area under the receiver operating curve (AUC) was lower for CASPI and CADPI, as compared to baseline $\mathrm{Pd} / \mathrm{Pa}(0.80$ vs. 0.82 vs. 0.89 , respectively), for predicting the FFR $\leq 0.80$. For a CASPI $<$ 0.88 there were no false positives with $100 \%$ specificity, and for a CASPI $>1.02$, there were no false negatives with $100 \%$ sensitivity. Similarly, for a CADPI $<0.8$ there were no false positives with $100 \%$ specificity, and for CADPI $>1.16$ there were no false negatives with $100 \%$ sensitivity. 